\title{
ANÁLISE DOS ASPECTOS LEGAIS DE PRESCRIÇÕES DE MEDICAMENTOS ANTIMICROBIANOS RETIDAS EM UMA DROGARIA NO MUNICÍPIO DE CARUARU-PE
}

T. R. R. TIMÓTEO ${ }^{1}$, C. G. de MELO ${ }^{1}$, C. S. B. AGUILERA ${ }^{1}$, L. P. SIQUEIRA ${ }^{1}$, L. C. P. B. B. SILVA ${ }^{1}$, E. O. SILVA ${ }^{1}$, V. A. W. SALES ${ }^{1}$, M. L. R. NEVES ${ }^{1}$, J. M. A. L. SANTOS $^{2}$, I. A. B. S. ALVES ${ }^{3}$.

${ }^{1-}$ Universidade Federal de Pernambuco, Centro de Ciências da Saúde, Departamento de Ciências Farmacêuticas.

${ }^{2-}$ Escola Técnica Regional, Caruaru-PE.

3- Universidade Federal de Pernambuco, Centro Acadêmico do Agreste, Núcleo de Ciências da Vida.

\section{E-mail para contato: camilagmmelo@gmail.com}

RESUMO - A prescrição é uma ferramenta de grande importância para o uso racional de medicamentos, pois consiste em fornecer as informações adequadas para a sua utilização. $O$ objetivo desse estudo foi analisar os aspectos legais das prescrições de medicamentos antimicrobianos retidas em uma drogaria no município de Caruaru-PE. Foi realizado um estudo transversal descritivo, averiguando os dispositivos legais necessários e indicados na RDC $n^{\circ}$ 20/2011. Foram analisadas 876 prescrições no período de janeiro a dezembro de 2015. A maioria das prescrições, 72,9\%, apresentou todas as informações referentes ao prescritor, porém apenas $2,51 \%$ abrangeram todos os dados referentes ao paciente. Das prescrições analisadas, 27,4\% apresentavam-se ilegiveis e 4,33\% continham rasuras. A ausência de dados de dispositivos legais de presença obrigatória nas prescrições de medicamentos antimicrobianos foi observada com significativa frequência no período de estudo. Com isso faz-se necessário instituir estratégias voltadas para educação e esclarecimento dos profissionais prescritores.

Palavras-chave: Prescrição, aspectos legais, uso racional de medicamento.

ABSTRACT - Prescription is a very important tool for the rational use of medicines because it provides adequate information for their use. The aim of this study was to analyze the legal aspects and the profile of antimicrobial drug prescriptions retained in a drug store in the city of Caruaru, PE. A descriptive cross-sectional study was carried out, checking the profile of the prescriptions and the necessary legal provisions that are indicated in the RDC 20/2011. A total of 876 prescriptions was analyzed between January and December 2015. Most of the prescriptions, 72.9\%, presented all the information about the prescriber, but only $2.51 \%$ covered all data related to the patient. Among the prescriptions analyzed, 27.4\% were illegible and $4.33 \%$ were scribbled. The absence of mandatory data in prescriptions of antimicrobial drugs was found to be significantly relevant during the study period. Therefore, it is necessary to establish strategies aiming a better education and elucidation for the prescribers.

Keywords: Prescription, Legal aspects, Rational use of medicines. 


\section{INTRODUÇÃO}

A prescrição é um fator de grande importância para o uso racional do medicamento (LASTE et al., 2013). De acordo com a Portaria GM/MS 3.916/98 e a Portaria SVS/MS $344 / 98$, é o ato de definir o medicamento a ser consumido pelo paciente, apresentando informações necessárias como dosagem, duração de tratamento e orientação de uso (CRUCIOL-SOUZA et al., 2008; MASTROIANNI, 2009; SILVÉRIO; LEITE, 2010; NASCIMENTO; MAGALHÃES, 2013). As prescrições medicamentosas que não exibem todas as informações necessárias, que são ilegíveis e que apresentam rasuras, impedem a eficiência da dispensação gerando um risco à qualidade da assistência farmacêutica ao paciente, comprometendo o tratamento farmacoterapêutico e podendo ocasionar sérios danos ao mesmo (FERRARI et al., 2013).

No Brasil, a Lei ${ }^{\circ}$. 5.991/73 e o Decreto $n^{\circ}$. 74.170/74 dispõem sobre o controle sanitário do comércio de drogas, medicamentos, insumos farmacêuticos e correlatos. De acordo com essa legislação e também de acordo com a RDC n ${ }^{\circ} 20 / 2011$, as informações referentes ao prescritor, ao paciente e ao tratamento empregado devem estar incluídas na prescrição (BRASIL, 2011). Tendo como exemplo, a prescrição deve ser legível, informando data de emissão, descrição do medicamento (DCB/DCI ou nome comercial), apresentação do medicamento, forma farmacêutica, modo de uso, nome e endereço do paciente, nome, endereço, assinatura e número de inscrição no respectivo conselho do profissional do prescritor (LASTE et al., 2013; BARREIRA et al., 2011).

Pesquisas evidenciam que mais de $50 \%$ das prescrições de antimicrobianos no mundo são inadequadas (ETIENNE et al., 2011; NICOLINI et al., 2008). Uma parcela significativa corresponde a um aumento do uso inapropriado de antimicrobianos, principalmente em situações nas quais estes não se aplicam, como em infecções virais, sendo utilizados em excesso, principalmente em crianças (ROCHA et al., 2012). Por conseguinte, verifica-se o aparecimento de microrganismos cada vez mais resistentes aos antimicrobianos (FIOL et., 2013).

O presente estudo tem como objetivo analisar os dispositivos legais das prescrições de medicamentos antimicrobianos retidas em uma farmácia no município de Caruaru-PE.

\section{MATERIAIS E MÉTODOS}

Foi realizado um estudo transversal descritivo em uma drogaria do município de Caruaru, onde foram analisadas 876 prescrições de antimicrobianos (retidas na drogaria), durante o período de janeiro a dezembro de 2015 .

Os dados foram obtidos através da análise das prescrições, averiguando os dispositivos legais necessários e indicados na RDC n 20/2011: informações referentes ao prescritor; informações referentes ao paciente; dose ou concentração; posologia; quantidade prescrita; forma farmacêutica; legibilidade de prescrição; presença de rasuras; data de emissão.

\section{RESULTADOS E DISCUSSÃO}

Após análise das informações necessárias referentes aos prescritores, observou-se que 99,2\% das prescrições apresentavam assinatura, 97,16\% carimbo, 90,91\% endereço e $74,4 \%$ telefone do prescritor, porém, $72,9 \%$ compreendiam todas as informações legais referentes ao prescritor. 
Com relação às informações referentes ao paciente, $100 \%$ das prescrições apresentavam nome do paciente, porém 93,61\% não apresentavam idade e 97,38\% não apresentavam sexo do paciente. Apenas 2,51\% (22) continham todas as informações legais necessárias referente ao paciente na prescrição, fato que demonstra 0 descumprimento ou o desconhecimento por parte dos prescritores acerca das informações obrigatórias nas prescrições de medicamentos antimicrobianos, dificultando a identificação do paciente e o processo de dispensação.

Sobre os dispositivos legais do medicamento, foi identificada a ausência de várias informações essenciais nas prescrições. Observa-se que $86,07 \%$ informavam a dosagem ou concentração, assemelhando-se com o estudo realizado por Martins e colaboradores (2014), que observou 83,7\%. A posologia, a quantidade e a forma farmacêutica estiveram ausentes em $35,04 \%, 26,03 \%$ e $36,53 \%$ das prescrições analisadas, respectivamente.

Dificuldades na leitura e entendimento da informação escrita na prescrição podem aumentar o índice de erros de medicação. Das prescrições analisadas, 27,4\% apresentavam-se ilegíveis, $4,33 \%$ continham rasuras.

A data é uma informação relevante, pois esta assegura a validade da prescrição que, de acordo com a RDC n ${ }^{\circ}$ 20/2011, é de 10 dias após a data de emissão. No presente estudo, $14,39 \%$ das prescrições analisadas não apresentavam data de emissão. Esta característica da validade da prescrição é de grande importância, pois impõe um limite de dias para a dispensação do medicamento, forçando o paciente a adquirir o tratamento em um espaço de tempo menor, possibilitando uma terapêutica eficaz.

\section{CONCLUSÃO}

O elo entre médico, paciente e farmacêutico é respaldado pela prescrição medicamentosa. E para que ocorra uma comunicação adequada é necessário que a prescrição seja transmitida de forma persuasiva e clara. A ausência de dados de dispositivos legais de presença obrigatória nas prescrições de medicamentos antimicrobianos foi observada com significativa frequência no período de estudo. Este fato coloca em risco a dispensação do medicamento, a qualidade da assistência farmacêutica e o tratamento farmacoterapêutico. Portanto, é de grande importância que os problemas pertencentes à prescrição medicamentosa, principalmente em relação aos dispositivos legais essenciais para a dispensação, sejam minimizados, mediante o esclarecimento da indispensabilidade de estratégias voltadas para educação e esclarecimento dos profissionais prescritores.

\section{REFERÊNCIAS}

BARREIRA, P.F. et al. Prescrições medicamentosas: luz ou sombra para o usuário e farmacêutico. Rev. Bras. farm. v. 92, n. 4, p. 340-345, 2011.

BRASIL. Ministério da Saúde. Portaria SVS/MS n 20, 5 de maio de 2011. Resolução Colegiada da Agência Nacional da Vigilância Sanitária. 2011.

CRUCIOL-SOUZA, J.M.; THOMSON, J.C.; CATISTI, D.G. Avaliação de prescrições medicamentosas de um hospital universitário brasileiro. Rev. Bras. Educ. Med. v. 32, p. 188-96, 2008.

ÉTIENNE, P. et al. Antimicrobial stewardship program and quality of antibiotic prescriptions. Médecine et maladies infectieuses. v. 41, p. 608-612, 2011. 
FERRARI, C.K.B. et al. Falhas na Prescrição e Dispensação de Medicamentos Psicotrópicos: Um problema de Saúde Pública. Rev. Ciênc. Farm. Básica. Apl. v. 34, p. 109-116, 2013.

FIOL, F.S.D. et al. Evaluation of the prescription and use of antibiotics in Brazilian children. The Brazilian Journal of Infectious Deseases. v.17, n.3, p.332-337, 2013.

LASTE, G. et al. Análise de prescrições médicas dispensadas em farmácia no sistema único de saúde. Revista HCPA. v. 33 (1), p. 15-25, 2013.

MASTROIANNI, P.C. Análise dos aspectos legais das prescrições de medicamentos. Rev. Ciênc. Farm. Básica Apl. v. 30 (2), p.173-176, 2009.

NASCIMENTO, P.S.; MAGALHÃES, I.R.S. Análise da prescrição de antimicrobianos dispensados em uma rede de drogarias da região Norte do Brasil. Rev. Bras. Farm. v.94 (3), p. 211-218, 2013.

NICOLINI, P. et al. Fatores relacionados à prescrição médica de antibióticos em farmácia pública da região Oeste da cidade de São Paulo. Ciênc. Saúde Col. v. 13, p. 689-696, 2008.

ROCHA, M.C.P. et al. Perfil de prescritores e prescrição de antimicrobianos nas infecções das vias aéreas superiores em Pediatria. Rev Paul Pediatr. v. 30 (4), p. 471478, 2012.

SILVÉRIO, M.S.; LEITE, I.C.G. Qualidade das prescrições em município de Minas Gerais. Rev. Assoc. Med. Bras. v. 56, n.6, p. 675-80, 2010. 